on an extensive table of values of a puir of numerical functions. Although the table is only partially completed, I have shown by this method that, aside from those already known, there are no factors under $524,288,000$ of any $F_{n}, n>16$, as against the limit 100,000,000 given by Cunningham and Western.* Incidentally, this method shows four errors in a list of forty-five numbers of the form $Q_{n}$ studied by Seelhoff. $\uparrow$

New Haven, Conn., June $15,1905$.

\title{
SIMPLY TRANSITIVE PRIMITIVE GROUPS WHICH ARE SIMPLE GROUPS.
}

BY PROFESSOR H. L. RIETZ.

(Read before the Chicago Section of the American Mathematical Society, April 22, 1905).

IN the papers published by several writers $\$$ relating to the existence of simple groups of odd composite order, much use is made of the fact that, if such a group existed, it could be represented as a simply transitive primitive substitution group. But this work has thus far led to no simple groups.

In working on this problem, it appeared well to examine some simply transitive primitive groups which are simple groups of even order, but upon examining the literature of primitive groups with a view to finding groups of this type, I find only two such groups. It therefore seems worth while to call attention to a system of groups of this type. This will be done by proving the following theorem :

There is a simple group $G$ of composite order corresponding to every prime number $p>11$ (where $p=2 q+1, q$ being a prime number) which can be represented as a simply transitive primitive group of degree

$$
1+\kappa p \quad\left(\frac{(p-2) !-1}{p} \geqq \kappa>1\right) .
$$

* Proc. Lond. Math. Soc. (2), vol. 1 (1904), p. 175.

†Zeitschrift für Math. $u$. Phys., vol. 31, p. 380. The present range of my table covers only eighteen numbers of Seelhoff's list.

† Miller, Proc. Lond. Math. Soc., vol. 33, pp. 6-10. Burnside, Proc. Lond. Math. Soc., vol. 33, pp. 162-185. Kietz, Amer. Journ. Math., vol. 26, pp. $1-30$. 
Select $G$ as a primitive group of degree $p$ containing more than one subgroup of order $p$, and such that its order $g \leqq g_{1}$, where $g_{1}$ is the order of any other primitive group of degree $p$ which contains more than one subgroup of order $p$. When $G$ is thus selected, it is easily shown to be a simple group. Any subgroup $P$ of order $p$ contained in $G$ is transformed into itself by $p q$ operations, and by Sylow's theorem

$$
g=p q(1+\kappa p) .
$$

By another theorem due to Sylow, $\kappa>1$ (Videnskabs-Selskabets Skriften, 1897, No. 9). The theorem referred to states that, when $p>11$, there is no primitive group of degree $p$ of order

$$
\frac{p(p-1)(p+1)}{2} \text {. }
$$

The $p q$ substitutions which transform $P$ into itself form a maximal subgroup of $G$ on account of the restriction on the order of $G$. With reference to this subgroup, $G$ could then be represented as a primitive group of degree $1+\kappa p$. Denote this representation by $G^{\prime}$. It remains to show that $G^{\prime}$ is simply transitive. This will be done by showing that its subgroup $G_{1}^{\prime}$ leaving a given letter fixed is intransitive. If $G_{1}^{\prime}$ were transitive, its degree $\kappa p$ would be an exact divisor of its order $p q$. Hence $\kappa p=p q$, since $\kappa>1 . \quad G_{1}^{\prime}$ would then be a regular group, and $G^{\prime}$ would contain in addition to the identical operation only substitutions of degrees $1+\kappa p$ and $\kappa p$. But Frobenius has proved * that a transitive group of degree $n$ and class $n-1$ contains an invariant subgroup made up of its substitutions of degree $n$ and identity. Hence $G^{\prime}$ would contain an invariant subgroup of order $1+\kappa p$. But this would be contrary to the hypothesis. Hence $G^{\prime}$ is a simply transitive primitive representation of the group. The upper limit of $\kappa$ is at once fixed from the way in which $G$ is selected.

The UNIVERSITY OF ILLINOIS.

* Berliner Sitzungsberichte, 1901, p. 1226. 